Int. J. Electrochem. Sci., 14 (2019) 3011 - 3023

\title{
The Micro Network of Polyacrylonitrile (PAN)-polyaniline (Pani)-graphene (GRA) Hybrid Nanocomposites for Effective Electrochemical Detection of Glucose and Improved Stability
}

\author{
Zupeng Yan ${ }^{1}$, Hao Zheng ${ }^{1, *}$, Jianfang Chen ${ }^{1,2}$, Ying Ye ${ }^{3}$ \\ ${ }^{1}$ Institute of Marine Chemistry and Environment, Ocean College, Zhejiang University, Zhoushan \\ 316021, People's Republic of China \\ ${ }^{2}$ Key Laboratory of Marine Ecosystems and Biogeochemistry, Second Institute of Oceanography, State \\ Oceanic Administration, Hangzhou 310012, People's Republic of China \\ ${ }^{3}$ Ocean College, Zhejiang University, Zhoushan 316021, People's Republic of China \\ *E-mail: zhenghao@ zju.edu.cn
}

doi: $10.20964 / 2019.03 .42$

Received: 27 November 2018 / Accepted: 7 January 2019 / Published: 7 February 2019

\begin{abstract}
A glucose biosensor was developed by immobilizing glucose oxidase (GOD) into the micro network of polyacrylonitrile (PAN)-polyaniline (Pani)-graphene (GRA) hybrid nanocomposite fabricated through phase inversion process. PAN with molecular weight of $\mathrm{M}_{\mathrm{w}}$ around $2.93 \times 10^{4}$ was synthesized by single rare-earth catalyst-Y(OAr $)_{3}$ and GRA with few-layers was prepared by electrochemical expansion of graphite in propylene carbonate electrolyte, respectively. The morphologies of nanocomposites and the fabricated process of biosensor were performed by scanning electron microscopy (SEM). The cyclic voltammetry (CV) was employed to evaluate electrochemical performance of the as-prepared biosensor. The apparent activation energy (Ea) of enzyme-catalyzed reaction based on Arrhenius equation was estimated to be $16.21 \mathrm{~kJ} \mathrm{~mol}^{-1}$. The constructed glucose biosensor exhibited a short response time within $5 \mathrm{~s}$, and a superior storage stability of preserving $96.28 \%$ of the original response over a period of 2 weeks and $91.95 \%$ of that even after a month. The linear range, sensitivity, detection limit, anti-interference, and practical application were also investigated. The micro network of PANPani-GRA hybrid nanocomposite provides a hopeful candidate for construction of biosensors.
\end{abstract}

Keywords: Polyacrylonitrile; Polyaniline; Graphene; Glucose biosensor.

\section{FULL TEXT}

(C) 2019 The Authors. Published by ESG (www.electrochemsci.org). This article is an open access article distributed under the terms and conditions of the Creative Commons Attribution license (http://creativecommons.org/licenses/by/4.0/). 